\title{
Effect of Multiple Liquid Inlets on Mass Transfer in Rotating Packed Beds
}

\author{
Jakub Elcner ${ }^{1, *}$, and Miroslav Jicha ${ }^{1}$ \\ ${ }^{1}$ Brno University of Technology, Faculty of Mechanical Engineering, Department of \\ Thermodynamics and Environmental Engineering, Technicka 2986/2, 616 69, Brno, Czech Republic
}

\begin{abstract}
This study deals with optimalization of rotating packed bed design using computational fluid dynamics approach. Comparison of three variants of liquid distributor were performed on 2D geometry. Turbulence was modelled using unsteady RANS approach and volume of fluid technique were used to simulate gas-liquid interphase. Results were compared on basis of liquid holdup evaluation.
\end{abstract}

\section{Introduction}

Carbon dioxide $\mathrm{CO}_{2}$ contributes with approximately $60 \%$ to global warming. If we leave aside sustainable sources, then the technology CCS (Carbon Capture and Storage) becomes key strategy for reduction of $\mathrm{CO}_{2}$ from fossil fuels. In the complete system of CCS technology the most expensive is the $\mathrm{CO}_{2}$ capture that represents about $70 \%$ of the total cost for required $90 \% \mathrm{CO}_{2}$ reduction. Research and development focuses on intensification of the capture process. According to many studies the most advanced and mature technology when comparing with other methods is chemical absorption, which is on the Technology Readiness Level 9 (TRL9), and also on the solvent regeneration side. Absorption is carried out in various apparatuses, the most commonly used are static columns, which are filled with different types of packings. However the static columns or any spray systems like scrubbers are in principle limited by gravitation and the process cannot be further intensified. In addition, static columns are extremely large vessels, often over 30 meters in height and large in diameter, which leads to high investment cost and space.

Therefore, the aim is to intensify the absorption process. This can be reached by using a rotary absorber called Rotating Packed Bed (RPB). Rotary absorber can develop up to twenty times the gravitational acceleration and subsequently ensure up to one order of magnitude higher transfer of $\mathrm{CO}_{2}$ to the solvent. All this with dimensions ten times smaller than when using static columns or scrubbers. The main task in the development of RPB is to clarify the influence of geometry, rotational speed, gas inlet, primary solvent distribution, and type of packing (wire mesh or metal foam) on liquid distribution, and liquid disintegration and film formation, i.e. phase interface inside rotating packing. The most suitable way to assess the influence of various parameters and to propose an optimal solution for RPB is to use computational fluid dynamics to simulate the process

*Corresponding author: elcner@fme.vutbr.cz 
inside the packing and validate it by lab scale experiments. The efficiency of $\mathrm{CO}_{2}$ capture is evaluated on the basis of a parameter called liquid holdup, i.e. an amount of solvent that is retained inside the packing and forms an interphase between solvent and gas.

\section{Methods}

\subsection{Model}

For comparison, a classic arrangement of a rotating packed bed, based on experiments of [1] and numerical simulations of [2], were selected. Three variants of geometry with identical wire packing and outlet placement but a different arrangement of liquid distributors located in the inner wall of the geometry were created. Arrangement of inlet distributors and their size for each variant of geometry was designed to achieve an equal distribution of liquid into the wired packing and hence maintain the same fluid velocity. The geometries can be seen in Figure 1. Each variant of the model contains wire mesh packing with wires of circular shape. The diameter of the wires is $0.5 \mathrm{~mm}$ and the distance between the centre of the adjacent wires in the circumferential direction is $3.5 \mathrm{~mm}$. The internal diameter of the packing is $42 \mathrm{~mm}$ and the outer diameter is $82 \mathrm{~mm}$. Packing contains 21 concentric layers in total, and the distance between two layers of wires in the radial direction is $1 \mathrm{~mm}$. The void fraction of the packing is 0.96 . Liquid outflow was provided by ten outlets of the size of $3 \mathrm{~mm}$, evenly distributed on the outer wall.

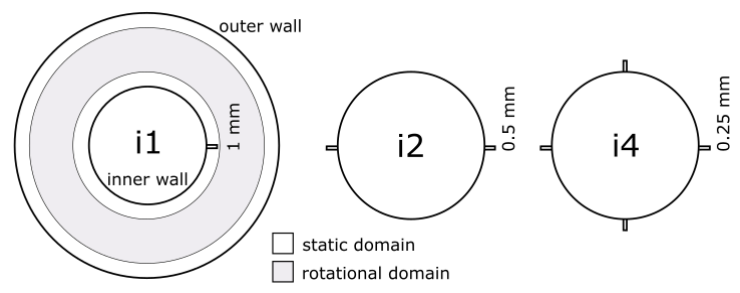

Fig. 1. Variants of geometry with different amount of liquid distributors.

A two-dimensional computational grid with unstructured quadrilateral elements with the average size of elements $0.00015 \mathrm{~m}$ was generated. A prismatic layer consisting of 8 layers were generated on the inner, outer wall, and wires of packing for better treatment of nearwall boundary layer. The average size of the grid for all variants of geometry was 550000 of cells. Details of the computational grid (inlet and wires on the variant i1) can be seen in Figure 2.

Fig. 2. Detail of computational grid.

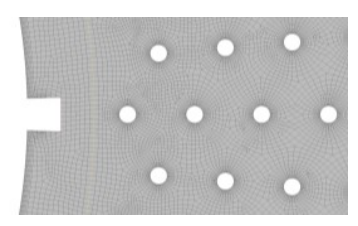

\subsection{Numerical setting}

Numerical calculations were performed using Simcenter Star-CCM + solver in version 2019.2 build 14.04.011. Assumptions for calculations were that the liquid and gas phase were both Newtonian and incompressible with no phase change and the whole process is 
isothermal. Surface tension between these phases is constant and uniform and its value is $0.0728 \mathrm{~N} / \mathrm{m}$. Interaction between gas (air: density $=1.18415 \mathrm{~m}^{3} / \mathrm{kg}$; dynamic viscosity = $1.85508 \mathrm{E}-5 \mathrm{~Pa} / \mathrm{s}$ ) and liquid (water: density $=998.2 \mathrm{~m}^{3} / \mathrm{kg}$; dynamic viscosity $=1.003 \mathrm{E}-$ $3 \mathrm{~Pa} / \mathrm{s}$ ) phase was modelled using Volume of Fluid (VOF) multiphase flow model by tracking the gas-liquid interface. Because the breakup of the liquid during the investigated process requires high resolution of the computational grid and hence demands high computational power, the RPB had to be modelled on the two-dimensional domain. Turbulence was modelled using the unsteady RANS approach with SST k- $\omega$ model with the compressibility correction option activated. A time step was set to $1 \mathrm{E}-5 \mathrm{~s}$ with 10 inner iterations and simulation was calculated for $1.5 \mathrm{~s}$. The contact angle on the wires was set to $150^{\circ}$ which corresponds to a hydrophilic material, conversely, the contact angle on the walls of RPB was set to $30^{\circ}$ (hydrophobic material). Movement of the wire packing was modelled using a moving reference frame with the axis of rotation in the centre of the rotational domain and the rotational speed of $1000 \mathrm{rpm} .100 \%$ of the liquid phase was set the inlets of liquid located on the inner wall of the RPB. The velocity of the fluid at the inlets was $2 \mathrm{~m} / \mathrm{sec}$. The identical mass flow rate into the domain for all of the three variants of RPB was monitored using mass flow report and it was controlled by the dimension of inlet nozzle.

\subsection{Validation}

Calculations were validated using experimental data from [1], numerical simulations of [2], and with the correlation proposed by [3] Values of physical properties were set according to experiments of [1]. A comparison of the liquid holdup (surface average of volume fraction of water) can be seen in Figure 3.

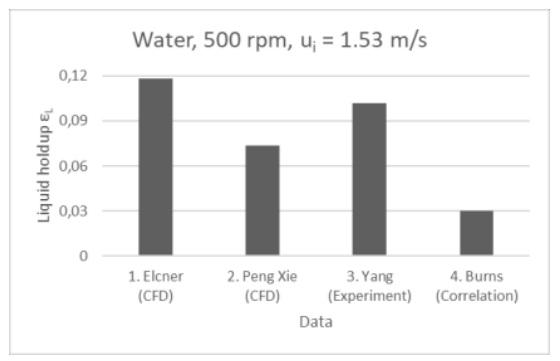

Fig. 3. Validation of numerical settings

The liquid holdup was estimated for the whole geometry (static and rotational domain) with one inlet. Our results appear to be overestimated in comparison with the experiments while the calculations of [2], underestimated it. The overall match with experiments is slightly better for our calculations.

\section{Results and discussion}

Results were compared based on liquid holdup estimated for the packing area (rotational domain) to neglect the influence of the liquid agglomeration on outer walls of the domain. Figure 4 shows the result for $1.5 \mathrm{~s}$ of phenomena. 


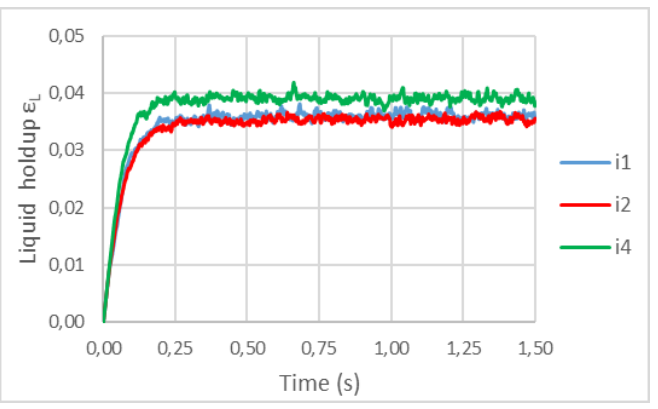

Fig. 4. Comparison of liquid holdup for all variants of liquid distribution.

The influence of the number of liquid distributors on the liquid holdup can be seen from the results. It takes $0.25 \mathrm{~s}$ to initiate and stabilize the course of liquid holdup values. After this time, a solution can be considered to be stable with small oscillations. While the cases with one (i1) or two (i2) liquid distributors evinced minimal differences in liquid holdup during the intensification process, the case with four distributors (i4) showed better performance. Whereas the same amount of water is delivered to the system, the differences in liquid holdup must be caused by the different number of liquid distributors. From the actual layout of water in the packing (Figure 5) can be seen that the area of liquid dispersion occurrence grows with the number of distributors, which has a positive effect on the liquid holdup values.
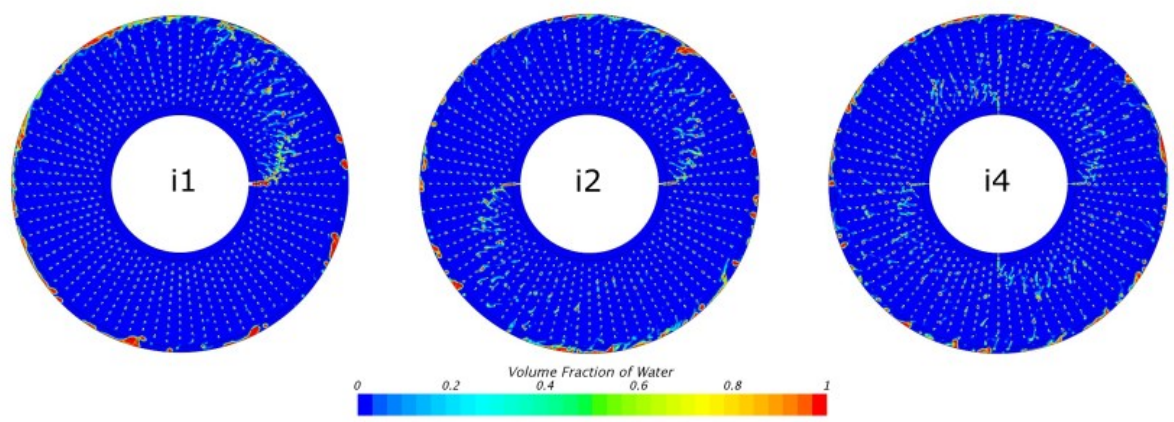

Fig. 5. The actual volume fraction of water for $t=1.5 \mathrm{~s}$.

Nonetheless, this was only an initial study on the liquid distributor number and its influence on the liquid holdup. Since it was performed in 2D and it has some simplifying assumption mentioned above, it needs to be confirmed during the next research.

This work was financially supported by the project "Computer Simulations for Effective LowEmission Energy" funded as project No. CZ.02.1.01/0.0/0.0/16_026/0008392 by Operational Programme Research, Development and Education, Priority axis 1: Strengthening capacity for highquality research. The financial support is gratefully acknowledged.

\section{References}

1. Y. C. Yang, Y. Xiang, G. W. Chu, H. K. Zou, Y. Luo, M. Arowo, J. F. Chen, Chem. Eng. Sci. 138 (2015)

2. P. Xie, X. Lu, X. Yang, D. Ingham, L. Ma, M. Pourkashanian, Chem. Eng. Sci. 172 (2017)

3. J. R. Burns, J. N. Jamil, C. Ramshaw, Chem. Eng. Sci. 55 (2000) 